\title{
Malabsorption of antimycobacterial drugs as a cause of treatment failure in tuberculosis
}

\author{
João Bento, ${ }^{1}$ Raquel Duarte, ${ }^{2,3}$ Maria Céu Brito, ${ }^{3}$ Sónia Leite, ${ }^{4}$ Maria Rosário Lobato, ${ }^{5}$ \\ Maria do Carmo Caldeira, ${ }^{5}$ Aurora Carvalho ${ }^{2,3}$ \\ ${ }^{1}$ Department of Pulmonology, Hospital de S Joao, Alameda Professor Hernani Monteiro, Porto, Portugal \\ ${ }^{2}$ Centro de Diagnóstico Pneumológico de Vila Nova de Gaia, Rua Conselheiro Veloso da Cruz, Vila Nova de Gaia, Portugal \\ ${ }^{3}$ Department of Pulmonology, Centro Hospitalar de Vila Nova de Gaia/Espinho, Rua Conceição Fernandes, Vila Nova de Gaia, Portugal \\ ${ }^{4}$ Department of Gastrenterology, Centro Hospitalar de Vila Nova de Gaia/Espinho, Rua Conceição Fernandes, Vila Nova de Gaia, Portugal \\ ${ }^{5}$ Lisbon Pharmacy Department, Lisboa, Portugal
}

Correspondence to João Bento, joao.a.p.bento@gmail.com

\section{Summary}

Malabsorption of oral antimycobacterial drugs is a rare cause of treatment failure in tuberculosis (TB). Several predisposing comorbidities have been recognised. HIV infection is the most important risk factor referred in the literature. There are few reports about antimycobacterial drugs malabsorption, particularly in the absence of predisposing comorbidities. The authors present a clinical case of oral treatment failure in TB due to malabsorption; however, what caused the failure remained unclear. Possible causes of malabsorption are discussed under various sections. Purpose of this case report is to point to this rare situation that can easily go unnoticed unless a very high level of suspicion is present.

\section{BACKGROUND}

Most patients with tuberculosis (TB) respond well to treatment. Treatment failure is still an ongoing challenge. Resistance and non-compliance to therapy are the most important causes. Even in patients with good compliance to directly observed therapy (DOT) and adequate sensitivity for the drugs in use, treatment failure still occurs and malabsorption of orally administered drugs is one possible explanation for this situation.

\section{CASE PRESENTATION}

Our case is a 15-year-old male student, who was obese (94 $\mathrm{kg}$ ), previously healthy and who presented with a 5 -month history of cough, sputum, anorexia and weight loss (10 kg).
He presented no vomits and his bowel history was normal, with no diarrhoea. He was taking no medication. He was non-smoker and had no alcohol or drug addiction. Clinical, radiological (figure 1) and microbiological evaluation supported the diagnosis of respiratory TB. Daily oral weightadjusted standard combination therapy (isoniazid (INH): $300 \mathrm{mg}$, rifampin (RIF): $600 \mathrm{mg}$, pyrazinamide (PZA): 1500 $\mathrm{mg}$ and ethambutol (EMB): $1200 \mathrm{mg}$ ) was started. Medication was taken in fasting or after a light snack, under DOT regime, and as witnessed by an experienced nurse. After taking medication, patient remained in our department under direct supervision for about $2 \mathrm{~h}$ to ensure that medication was not rejected or vomited or expelled. Patient's HIV status was negative. Sputum-culture exam confirmed

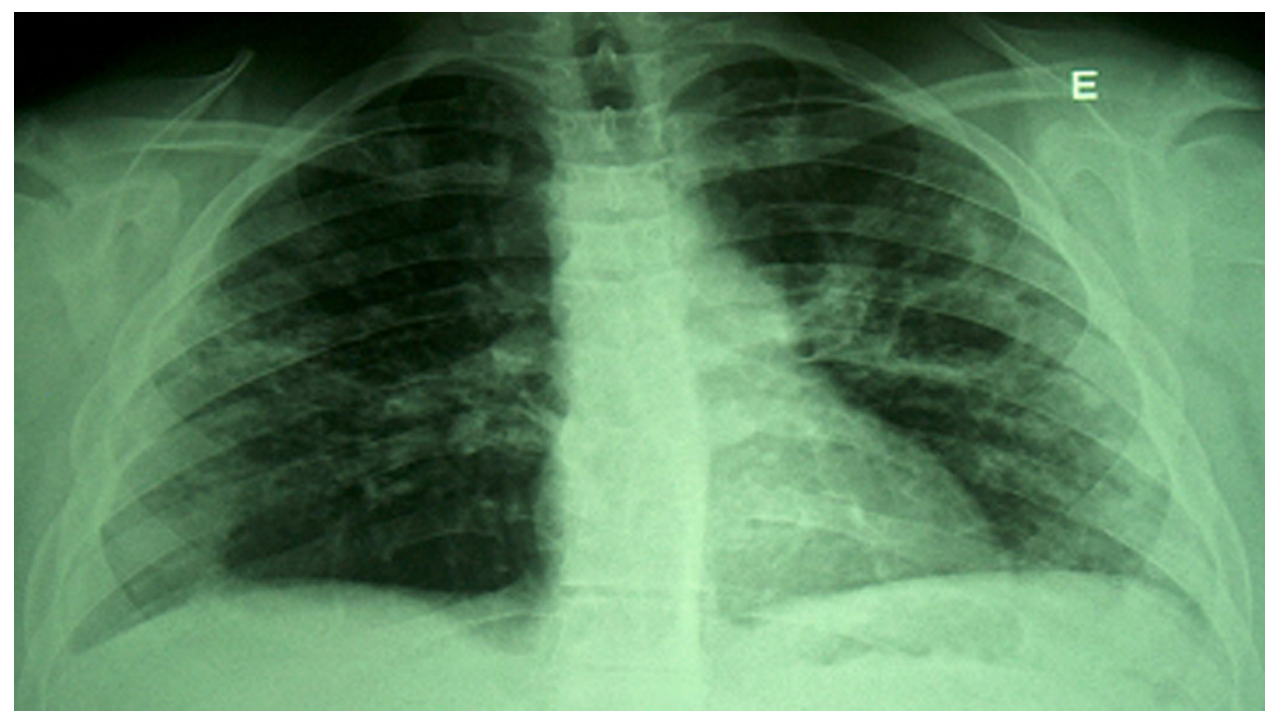

Figure 1 Chest x-ray (initial). Extensive bilateral infiltrate with a cavitary lung lesion on the left. 


\section{BMJ Case Reports}

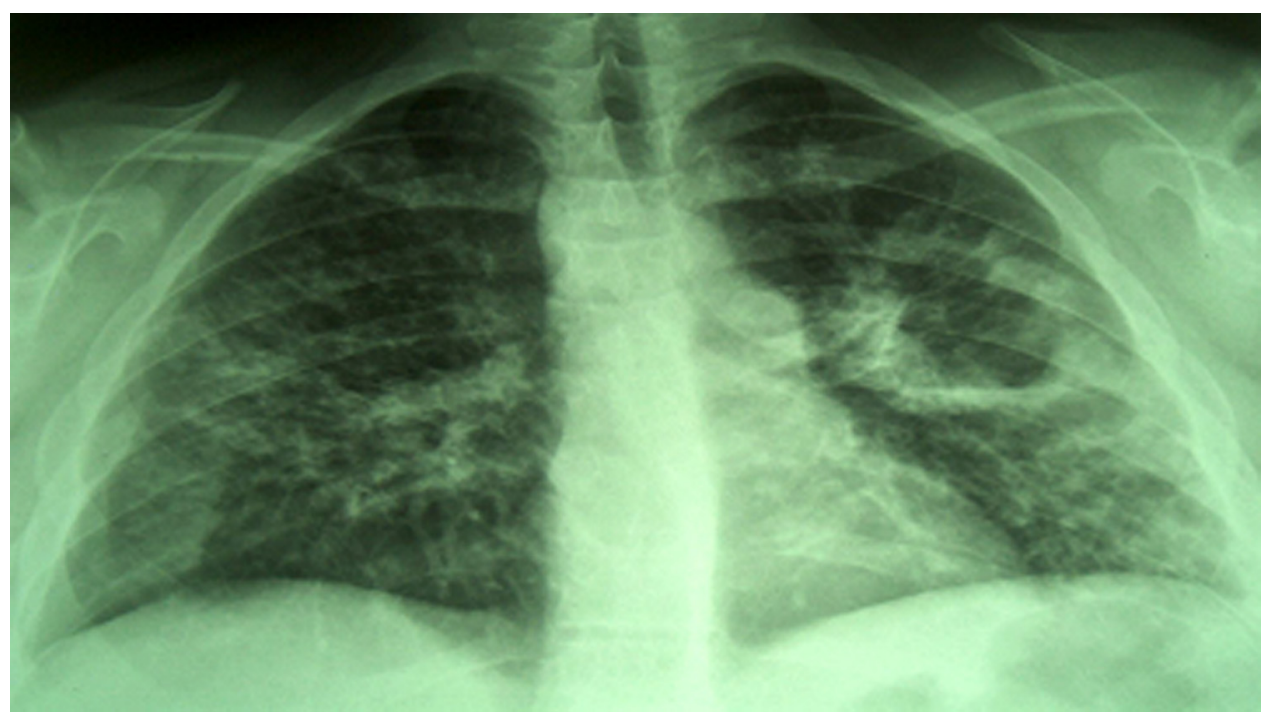

Figure 2 Chest x-ray (after 4 months of initial treatment). Worsening radiological pattern: more exuberant bilateral lung infiltrate and larger cavitary lung lesion on the left.

Mycobacterium tuberculosis (MT) sensitive for all first-line drugs in use. Nevertheless, patient failed to improve (clinical and radiologically (figure 2)) and monthly repeated sputum smear during first 4 months of treatment showed persistence of acid-fast bacilli (AFB).

\section{INVESTIGATIONS}

Drug-susceptibility testing (DST) was repeated, which confirmed sensitivity for all drugs in use. Oral drugs malabsorption was then considered as a cause for treatment failure, which was confirmed by measurement of anti-TB drugs blood levels before and after the beginning of parenteral TB drugs during the few days the patient was in the hospital. Serum concentration of INH was undetected $(2 \mathrm{~h}$ and $6 \mathrm{~h}$ postdose) and RIF level was undetected after $2 \mathrm{~h}$ and was below expected range $6 \mathrm{~h}$ postdose, which supported our hypothesis (table 1). Comorbidities that can impair drug absorption (HIV infection, hypoalbuminemia, gastrointestinal or malabsorptive diseases; infectious gastroenteritis, liver, renal or thyroid diseases) were excluded. In collaboration with Gastroenterology Department, a study for gastrointestinal malabsorption was conducted. Laboratorial evaluation, including assessing blood levels of folate, vitamin B12, iron, calcium, alkaline phosphatase and albumin, was normal. Serologic tests consisting on antiendomysial antibodies and antigliadin antibodies were normal. Stool studies were normal, including qualitative assessment of faecal fat, Sudan stain and assessment of enteric pathogens, cists, parasites and MT. The D-xylose test was normal. Gastrointestinal endoscopy with biopsy from the second part of the duodenum and abdominal ultrasound were also performed and revealed no changes.
Table 1 Blood levels of antimycobacterial drugs

\begin{tabular}{lllll}
\hline & \multicolumn{2}{l}{ INH blood level } & \multicolumn{2}{l}{ RIF blood level } \\
\hline Time postdosis & $\mathbf{2} \mathrm{h}$ & $\mathbf{6 h}$ & $\mathbf{2 h}$ & $\mathbf{6 h}$ \\
Treatment $A^{*}$ & n.d. & n.d. & n.d. & n.d. \\
Treatment $B^{* *}$ & n.d. & n.d. & n.d. & $\mathbf{0 . 3}$ ág/ml \\
Treatment $C^{* * *}$ & $\mathbf{8 . 9 6}$ ág/ml & 3.01 ág/ml & 5.73 ág/ml & 2.38 ág/ml
\end{tabular}

*Treatment A: INH $300 \mathrm{mg}$ oral, RIF $600 \mathrm{mg}$ oral, PZA $1500 \mathrm{mg}$ oral and EMB $1200 \mathrm{mg}$ oral.

** Treatment B: INH $450 \mathrm{mg}$ oral, RIF $700 \mathrm{mg}$ oral, PZA $2000 \mathrm{mg}$ oral and EMB $1800 \mathrm{mg}$ oral.

*** Treatment C: INH $300 \mathrm{mg}$ intravenous, RIF $600 \mathrm{mg}$ intravenous, PZA $2000 \mathrm{mg}$ oral and EMB $1800 \mathrm{mg}$ oral.(Therapeutic range: INH: $0.5-8.0 \mu \mathrm{g} / \mathrm{ml}$; RIF: 1.0-30 $\mu \mathrm{g} / \mathrm{ml}) \mathrm{EMB}$, ethambutol; INH, isoniazid; n.d., not detected; PZA, pyrazinamide; RIF, rifampin.

\section{TREATMENT}

Assuming the possibility of isolated malabsorption of antiTB drugs, patient was prescribed with intravenous INH, RIF and streptomycin (SM) while continuing intake of daily oral PZA and EMB. The DST by the beginning of this regime revealed resistance to SM, which was then suspended.

\section{OUTCOME AND FOLLOW-UP}

Meanwhile, patient began to improve clinically, 1 month after mycobacterial charge was significantly reduced and 2 months after he was asymptomatic and gaining weight. By that time chest $\mathrm{x}$-ray was improving and since then sputum smear became persistently AFB negative. Cultural exams turned to negative only after the third month of treatment. This patient had an extensive cavitary lung TB presenting good tolerance to treatment and no side effects, nor toxicity 


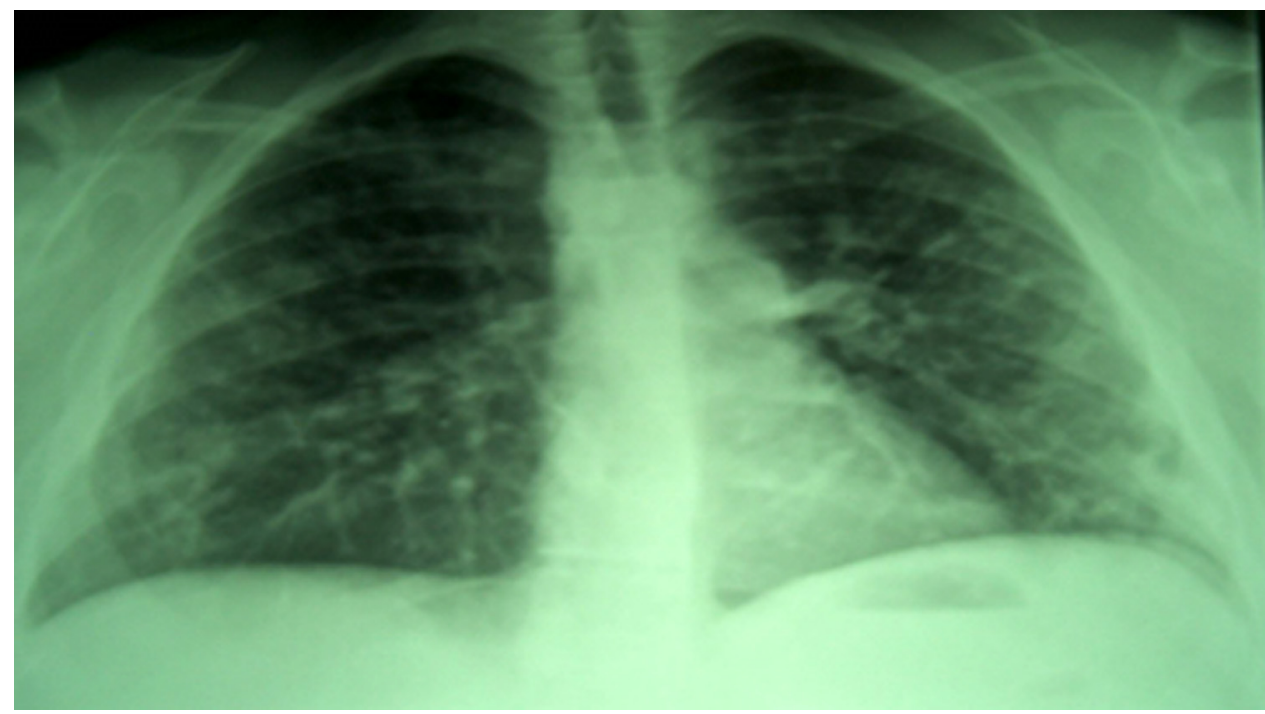

Figure 3 Chest $\mathrm{x}$-ray (after complete treatment). Almost complete chest $\mathrm{x}$-ray abnormalities resolution.

were detected. Tacking this in account our option was to extend the therapeutic regimen treating the patient for 9 months since culture exam have turned to negative, totalising 12 months of treatment. By that time patient was clinically well and chest $x$-ray was normal (figure 3). Patient has been kept in follow-up after treatment cessation and until now no signs (clinical or radiological) of relapse have been detected.

\section{DISCUSSION}

Oral malabsorption of anti-TB drugs is one possible cause for treatment failure, although rarely described. In patients receiving appropriate anti-TB therapy in DOT regime without clinical, radiological or microbiological improvement, malabsorption must be considered. ${ }^{1-6}$

Malabsorption of $\geq 1$ drug may justify treatment failure, adverse clinical outcome, disease progression and even promotion of drug resistance. ${ }^{12} 4578$

Most patients with TB absorb anti-TB drugs properly and do not require drug monitoring as long as they respond expectedly to therapy. ${ }^{19}$ Malabsorption of anti-TB drugs has been reported in a small subset of patients. ${ }^{1248}$ Potential causes have been recognised, although they remain poorly characterised. Most reports have focussed on HIV infection as the most important risk factor for anti-TB drugs malabsorption. ${ }^{1-5}$ 7-9 $\mathrm{HIV}$ patients may present lower serum concentrations of $\geq 1$ anti-TB drugs, even in the absence of malabsorption symptoms. ${ }^{17}$ Malabsorption in non-HIV patients is not clearly explained and data in literature are lacking. Other comorbidities that can possibly impair drug absorption include hypoalbuminemia in malnourished, gastrointestinal or malabsorptive diseases, infectious gastroenteritis, gastric achlorydria, end-stage liver or renal diseases. ${ }^{13}$

Altered drug bioavailability with the use of combination INH/RIF compared with individual tablet forms has also been reported in the literature. ${ }^{23}$ It is also known the deleterious effects of high-fat food on the absorption of $\mathrm{INH}$ and
RIF. So it is advisable that patients fasted before medication or restrict intake to a light snack.

Measurement of anti-TB drugs levels has been encouraged in patients infected with a sensitive MT, which remain AFB smear positive after 3 months of DOT regime with effective drugs according to DST. ${ }^{1-36}$ Maximum INH and RIF serum concentration are typically reached $2 \mathrm{~h}$ after oral administration and their normal half time is $2-4 \mathrm{~h}$. Two separate measurements (2-h and 6-h postdose) are advocated to distinguish between delayed absorption (late peak close to normal) and malabsorption (low concentrations at all time). ${ }^{6}$

This clinical case refers to a patient presenting respiratory TB not responsive to standard therapy. Despite good compliance to DOT regime and DST 3 months apart confirming sensitivity to first-line drugs in use, treatment failure has occurred.

To face the possibility of surreptitious non-adherence to treatment, our usual DOT regime was reinforced by maintenance of patient in our department after taking medication under direct supervision for about $2 \mathrm{~h}$ to ensure that medication was not rejected nor vomited or expelled.

Measurement of anti-TB drugs blood levels before and after the beginning of parenteral TB drugs was performed during a few days period of hospitalisation which revealed that serum concentration of INH and RIF were undetected or much below expected range, which supported our hypothesis of oral malabsorption of anti-TB drugs.

Patient's HIV status was negative. Gastrointestinal or malabsorptive disease, as a cause of drug malabsorption, seemed unlikely. Patient presented a good nutritional status, albumin and protein blood levels were normal and blood analysis revealed no signs of deficient absorption. Patient was not taking any antiacid medication. Moreover, a detailed study to clarify a possible gastrointestinal malabsorption was conducted in collaboration with Gastroenterology Department which revealed no abnormalities.

Gastrointestinal TB was excluded. However, according to literature, blood concentration of anti-TB drugs is not impaired in patients with gastrointestinal $\mathrm{TB} .^{10}$ 


\section{BMJ Case Reports}

In the absence of comorbidities pointed earlier, possibility of isolated anti-TB drugs malabsorption was considered. To clarify this situation, patient was admitted to pulmonology ward and measurement of anti-TB drugs levels was performed with patient taking oral anti-TB medication under a tighter DOT regime during the period of hospitalisation. Under these conditions, undetected or low serum concentration of anti-TB drugs strongly supported this hypothesis. Introduction of intravenous regime, followed by good clinical, radiological and microbiological response, confirmed malabsorption as the most possible cause for treatment failure.

\section{Learning points}

- For the vast majority of patients, standard drug regimes prescribed are adequate.

- Although rare, malabsorption of anti-TB drugs is an increasingly prevalent problem.

- There are few reports in the literature about TB treatment failure due to antimycobacterial drugs malabsorption and particularly in the absence of predisposing comorbidities.

- Nevertheless in patients who remain AFB smear positive after 3 months of adequate treatment under DOT regime and proven DST, drug blood levels monitoring must be considered.

- We believe that drug malabsorption as a cause for low drug blood concentrations and treatment failure applies to this clinical case, even if cause of malabsorption was not clear.

\section{REFERENCES}

1. Mehta JB, Shantaveerapa H, Byrd RP Jr, et al. Utility of rifampin blood levels in the treatment and follow-up of active pulmonary tuberculosis in patients who were slow to respond to routine directly observed therapy. Chest 2001; 120:1520-4.

2. Kimerling ME, Phillips $P$, Patterson $P$, et al. Low serum antimycobacterial drug levels in non-HIV-infected tuberculosis patients. Chest 1998;113:1178-83

3. Mcllleron H, Wash P, Burger A, et al. Determinants of rifampin, isoniazid, pyrazinamide, and ethambutol pharmacokinetics in a cohort of tuberculosis patients. Antimicrob Agents Chemother 2006;50:1170-7.

4. Peloquin CA, MacPhee AA, Berning SE. Malabsorption of antimycobacterial medications. N Engl J Med 1993;329:1122-3.

5. Patel KB, Belmonte R, Crowe HM. Drug malabsorption and resistant tuberculosis in HIV-infected patients. N Engl J Med 1995;332:336-7.

6. Peloquin CA. Therapeutic drug monitoring in the treatment of tuberculosis. Drugs 2002;62:2169-83.

7. Sahai J, Gallicano K, Swick L, et al. Reduced plasma concentrations of antituberculosis drugs in patients with HIV infection. Ann Intern Med 1997;127:289-93.

8. Pinheiro VGF, Ramos LMA, Monteiro HSA, et al. Intestinal permeability and malabsorption of rifampicin and isoniazid in active pulmonary tuberculosis. Braz J Infec Dis 2006;10:374-9.

9. Gurumurthy P, Ramachandran G, Hemanth Kumar AK, et al. Decreased bioavailability of rifampin and other antituberculosis drugs in patients with advanced human immunodeficiency virus disease. Antimicrob Agents Chemother 2004; 48:4473-5.

10. Vij JC, Govil A, Jain NK, et al. Bioavailability of rifampicin, isoniazid and pyrazinamide in patients with intestinal tuberculosis with malabsortion. Ind $\mathrm{J}$ Tub 1995;42:211-13.

\section{Competing interests None.}

Patient consent Obtained.

This pdf has been created automatically from the final edited text and images.

Copyright 2010 BMJ Publishing Group. All rights reserved. For permission to reuse any of this content visit

http://group.bmj.com/group/rights-licensing/permissions.

BMJ Case Report Fellows may re-use this article for personal use and teaching without any further permission.

Please cite this article as follows (you will need to access the article online to obtain the date of publication).

Bento J, Duarte R, Brito MC, Leite S, Lobato MR, Caldeira MdC, Carvalho A. Malabsorption of antimycobacterial drugs as a cause of treatment failure in tuberculosis. BMJ Case Reports 2010;10.1136/bcr.12.2009.2554, date of publication

Become a Fellow of BMJ Case Reports today and you can:

- Submit as many cases as you like

- Enjoy fast sympathetic peer review and rapid publication of accepted articles

- Access all the published articles

- Re-use any of the published material for personal use and teaching without further permission

For information on Institutional Fellowships contact consortiasales@bmjgroup.com

Visit casereports.bmj.com for more articles like this and to become a Fellow 